\title{
The Relationship between Cognitive Decline and Psychopathology in Patients with Schizophrenia and Bipolar Disorder
}

\author{
Moon-Doo Kim¹, Hye-Jin Seo ${ }^{2}$, Hyunju Yun ${ }^{3}$, Young-Eun Jung, ${ }^{1}$ Joon Hyuk Park ${ }^{1}$, Chang-In Lee', Ji Hyun Moon ${ }^{4}$, \\ Seong-Chul Hong ${ }^{5}$, Bo-Hyun Yoon ${ }^{3}$, Won-Myong Bahk ${ }^{2}$ \\ Departments of ${ }^{1}$ Psychiatry, ${ }^{4}$ Family Medicine, and ${ }^{5}$ Preventive Medicine, Jeju National University School of Medicine, Jeju, ${ }^{2}$ Department \\ of Psychiatry, College of Medicine, The Catholic University of Korea, Seoul, ${ }^{3}$ Department of Psychiatry, Naju National Hospital, Naju, Korea
}

\begin{abstract}
Objective: The primary goals of the present study were to assess intellectual function in participants with schizophrenia or bipolar disorder (BD) and to investigate the relationships between cognitive decline and the severity of each type of psychopathology.

Methods: The present study included 51 patients with schizophrenia and 42 with BD who were recruited from the psychiatry outpatient clinic of Jeju University Hospital between March 2011 and March 2014. The Korean Wechsler Adult Intelligence Scale (K-WAIS) was administered to each of the 93 participants, and they were categorized into two groups based on their current intelligence quotient $(I Q)$ and their estimated premorbid IQ: severely impaired group (SIG) and mildly impaired group (MIG). The Minnesota Multiple Personality Inventory (MMPI) and the Brief Psychiatric Rating Scale (BPRS) were used to assess psychopathology.

Results: The SIG schizophrenia participants exhibited significantly higher scores on the frequent (F) and schizophrenia (Sc) subscales of the MMPI, but significantly lower scores on the correction (K) and psychopathic deviate (Pd) subscales compared with the MIG schizophrenia participants. Furthermore, the BPRS scores were significantly higher in the SIG schizophrenia participants relative to the MIG schizophrenia participants. The SIG BD participants had significantly higher F, masculinity-femininity (Mf), paranoia $(\mathrm{Pa})$, and Sc but significantly lower Pd scores compared with the MIG BD participants.

Conclusion: The present findings revealed a significant discrepancy between the estimated premorbid levels of cognitive function and current cognitive function in participants with schizophrenia or BD. Moreover, this discrepancy was correlated with severity of psychopathology in both groups.
\end{abstract}

KEY WORDS: Schizophrenia; Bipolar disorder; Cognition; Psychopathology.

\section{INTRODUCTION}

Neurocognitive impairment represents a core symptomatic feature of schizophrenia and bipolar disorder. ${ }^{1)}$ In schizophrenia, these deficits persist throughout the course of the disease and may be predictive of various types of function. ${ }^{2}$ A similar but less severe pattern of cognitive impairment has also been observed in patients with bipolar disorder. ${ }^{3)}$ In the past, cognitive impairment in bipolar disorder patients was considered infrequent and limited to affective episodes. More recently, however, it has been

Received: March 19, 2015 / Accepted: March 24, 2015

Address for correspondence: Hye-Jin Seo, MD

Department of Psychiatry, The Catholic University of Korea,

Yeouido St. Mary's Hospital, 10 63-ro, Yeongdeungpo-gu, Seoul

150-713, Korea

Tel: +82-2-3779-1250, Fax: +82-2-780-6577

E-mail: carpe-diem80@daum.net shown that a majority of patients with bipolar disorder demonstrate high rates of functional and cognitive impairment even during periods in which there is a sustained remission of mood symptoms. ${ }^{4,5}$

Neurocognitive impairments substantially limit creativity, work performance, quality of life, self-esteem, and psychosocial functioning in patients with severe mental illnesses, especially schizophrenia. ${ }^{5)}$ Similarly, it is highly likely that sustained neurocognitive deficits critically contribute to major occupational, social, and interpersonal dysfunctions that are commonly observed in patients with bipolar disorder. ${ }^{6)}$

On the other hand, cognitive deficits may also contribute to the psychopathologies underlying symptoms in schizophrenia and bipolar disorder patients. Several cross-sectional studies have indicated that these patients' performance on neurocognitive tests is correlated with

(C) This is an Open-Access article distributed under the terms of the Creative Commons Attribution Non-Commercial License (http://creativecommons.org/licenses/by-nc/3.0) which permits unrestricted non-commercial use, distribution, and reproduction in any medium, provided the original work is properly cited. 
three major schizophrenic symptom types: positive, negative, or disorganized. ${ }^{7)}$ In fact, Albus et al. ${ }^{8)}$ reported that psychotic symptoms were the most influential variable affecting cognitive function in patients with bipolar disorder.

Therefore, the aims of the present study were to describe intellectual function in patients with schizophrenia or bipolar disorder and to investigate the relationships between intellectual decline and the severity of each type of psychopathology.

\section{METHODS}

\section{Participants}

For the present study, 51 participants with schizophrenia and 42 with bipolar disorder were recruited from the psychiatry outpatient clinic of Jeju University Hospital (Jeju, Korea) between March 2011 and March 2014. Patients were included in this study if they were diagnosed with either bipolar I or II disorder or schizophrenia according to the criteria of the Diagnostic and Statistical Manual of Mental Disorders, 4th edition (DSM-IV). The exclusion criteria were a diagnosis of an intellectual disability or neurological disorder, or a significant physical health problem that could interfere with cognitive function. All patients included in this study were clinically stable.

All 93 participants first completed the Korean Wechsler Adult Intelligence Scale (K-WAIS), and they were then divided into two groups according to the difference between their current intelligence quotient (IQ) and their estimated premorbid IQ: the severely impaired group (SIG) and mildly impaired group (MIG). SIG participants exhibited a score difference $\geq 15$ between their current IQ and estimated premorbid IQ, and the mildly impaired group had a score difference $<15$. The clinical characteristics of the participants were assessed using the Minnesota Multiple Personality Inventory (MMPI) and the Brief Psychiatric Rating Scale (BPRS). The present study was approved by the institutional review board of Jeju University Hospital in Jeju, Korea, and all subjects provided informed consent.

\section{Measures}

\section{K-WAIS}

The K-WAIS was used to estimate the IQ level of each participant. ${ }^{9)}$ This measure assesses three general areas of IQ (verbal IQ [VIQ], performance IQ [PIQ], and full-scale IQ) using six verbal subtests and five performance subtests: information, digit span, vocabulary, arithmetic, com- prehension, similarities, picture completion, picture arrangement, block design, object assembly, and digit symbol.

\section{Premorbid intelligence estimates}

The premorbid intelligence estimates were assessed using the three subtests of the standardized K-WAIS (VIQ, PIQ, and full-scale IQ), controlling for age and education. The premorbid IQ of each patient was inferred using the index of premorbid intelligence developed by Kim. ${ }^{10}$ )

\section{MMPI}

The MMPI is a screening instrument used to differentiate various forms of psychopathology. ${ }^{11)}$ This measure consists of 550 items across 10 clinical subscales and four validity subscales; the 10 clinical subscales detect various features of psychopathology including hypochondriasis (Hs), depression (D), hysteria (Hy), psychopathic deviate (Pd), masculinity-femininity (Mf), paranoia (Pa), psychasthenia ( $\mathrm{Pt}$ ), schizophrenia ( $\mathrm{Sc}$ ), hypomania (Ma), and social introversion $(\mathrm{Si})$, and the validity subscales consist of the cannot say, lie (L), frequent (F), and correction (K) scales.

\section{BPRS}

The BPRS was developed to evaluate patients' overall psychiatric status using a 7-point Likert scale. ${ }^{12)}$ This measure consists of 18 items covering a broad range of symptoms that are commonly seen during a psychotic relapse, including hallucinations, delusions, and disorganization, as well as mood disturbances that may also accompany a relapse.

\section{Statistical Analysis}

The demographic and clinical characteristics were analyzed with independent $t$-tests, chi-square tests, or Fisher's exact tests, as appropriate. Independent $t$-tests were also conducted to compare mean scores of the two groups on the IQ, MMPI, and BPRS. All statistical analyses were conducted using IBM SPSS Statistics for Windows (version 20.0; IBM Co., Armonk, NY, USA), and a $p$ value $<0.05$ was considered to indicate statistical significance.

\section{RESULTS}

\section{Demographic Characteristics}

The mean ages in the schizophrenia and bipolar disorder groups were $32.67 \pm 11.38$ years and $30.81 \pm 9.57$ years, respectively. There were no significant differences 
between the groups in sex, age, marital status, education, or socioeconomic status (Table 1).

\section{Clinical Characteristics}

The clinical data for the schizophrenia and bipolar disorder groups are summarized in Table 2. There were no significant differences between the two groups in terms of age of onset, duration of illness, number of admissions, or family psychiatric history.

\section{Current $I Q$ and Premorbid IQ}

The results of the $t$-tests used to compare IQ scores are presented in Table 3. Participants with bipolar disorder had significantly higher current scores on the verbal, performance, and full-scale IQ tests compared with participants with schizophrenia. Additionally, participants with schizophrenia had significantly lower scores on the vocabulary, comprehension, block design, and digit symbol subtests compared with participants with bipolar disorder. The bipolar group displayed higher premorbid IQ scores than the schizophrenia group, but the difference was not statistically significant.

\section{Subgroup Analyses of Schizophrenia and Bipolar Disorder}

A decrease of $\geq 15$ points in IQ from premorbid levels was observed in $52.9 \%$ of participants with schizophrenia and $31 \%$ of participants with bipolar disorder; these participants were classified as SIG. There was a significantly

Table 1. Characteristics of the participant groups

\begin{tabular}{lcc}
\hline \multirow{2}{*}{ Characteristic } & \multicolumn{2}{c}{ Group } \\
\cline { 2 - 3 } & $\begin{array}{c}\text { Schizophrenia } \\
(n=51)\end{array}$ & $\begin{array}{c}\text { Bipolar disorder } \\
(n=42)\end{array}$ \\
\hline Sex & $29(56.9)$ & $19(45.2)$ \\
Male & $22(43.1)$ & $23(54.8)$ \\
Female & $32.67 \pm 11.375$ & $30.81 \pm 9.567$ \\
Age (yr) & & \\
Marital status & $43(84.3)$ & $25(59.5)$ \\
Married & $5(9.8)$ & $17(40.5)$ \\
Unmarried & $3(5.9)$ & $0(0.0)$ \\
Separation or divorce & $1(2.0)$ & $1(2.4)$ \\
Education (yr) & $25(49.0)$ & $10(23.8)$ \\
$7-9$ & $24(47.1)$ & $30(71.4)$ \\
10-12 & $1(2.0)$ & $1(2.4)$ \\
13-16 & & $1(2.4)$ \\
$\geq 16$ & $2(3.9)$ & $41(97.6)$ \\
Socioeconomic status & $46(90.2)$ & $0(0.0)$ \\
High & $3(5.9)$ & \\
Middle & &
\end{tabular}

Values are presented as number (\%) or mean \pm standard deviation. higher number of participants with severe intellectual decline in the schizophrenia group than in the bipolar disorder group $\left(\chi^{2}=4.543, p=0.038\right)$.

\section{MMPI and BPRS Scores in the Intellectual Subgroups of the Schizophrenic Participants}

The SIG schizophrenia participants exhibited significantly higher scores on the F and Sc subscales of the MMPI, but significantly lower scores on the $\mathrm{K}$ and $\mathrm{Pd}$ subscales than did the MIG schizophrenia participants (Table 4). Moreover, the BPRS scores were significantly higher in the SIG schizophrenia participants.

\section{MMPI and BPRS Scores in the Intellectual Subgroups of the Bipolar Disorder Participants}

The SIG bipolar disorder participants had significantly

Table 2. Clinical characteristics of the participant groups

\begin{tabular}{lcc}
\hline \multirow{2}{*}{ Characteristic } & \multicolumn{2}{c}{ Group } \\
\cline { 2 - 3 } & Schizophrenia & Bipolar disorder \\
\hline Age of onset (yr) & $29.25 \pm 10.88$ & $29.45 \pm 9.92$ \\
Duration of illness (yr) & $3.47 \pm 2.52$ & $3.26 \pm 1.91$ \\
Number of admissions & $2.35 \pm 2.11$ & $2.26 \pm 1.81$ \\
Family psychiatry history & $11(21.6)$ & $7(16.7)$ \\
Yes & $40(78.4)$ & $35(83.3)$ \\
No & &
\end{tabular}

Values are presented as mean \pm standard deviation or number (\%).

Table 3. Comparisons of intelligence quotient (IQ) levels in the participant groups

\begin{tabular}{|c|c|c|c|}
\hline \multirow{2}{*}{ Variable } & \multicolumn{2}{|c|}{ Group } & \multirow{2}{*}{$p$ value } \\
\hline & Schizophrenia & Bipolar disorder & \\
\hline \multicolumn{4}{|l|}{ IQ level } \\
\hline Verbal & $96.06 \pm 13.50$ & $101.40 \pm 11.79$ & 0.047 \\
\hline Performance & $91.33 \pm 12.10$ & $98.64 \pm 14.72$ & 0.010 \\
\hline Full scale & $93.53 \pm 12.30$ & $99.69 \pm 12.65$ & 0.020 \\
\hline \multicolumn{4}{|l|}{ Subtest } \\
\hline Information & $9.73 \pm 2.80$ & $9.50 \pm 2.31$ & 0.677 \\
\hline Digit span & $10.25 \pm 3.29$ & $10.86 \pm 2.58$ & 0.336 \\
\hline Vocabulary & $8.65 \pm 2.33$ & $10.17 \pm 2.61$ & 0.004 \\
\hline Arithmetic & $8.69 \pm 2.83$ & $9.29 \pm 2.96$ & 0.322 \\
\hline Comprehension & $9.57 \pm 2.95$ & $10.88 \pm 2.73$ & 0.030 \\
\hline Similarities & $9.69 \pm 2.61$ & $10.02 \pm 2.37$ & 0.520 \\
\hline Picture completion & $7.65 \pm 2.51$ & $8.21 \pm 2.78$ & 0.305 \\
\hline Picture arrangement & $8.71 \pm 2.48$ & $9.71 \pm 2.75$ & 0.067 \\
\hline Block design & $8.67 \pm 2.77$ & $10.12 \pm 3.13$ & 0.020 \\
\hline Object assembly & $9.29 \pm 2.71$ & $9.88 \pm 2.51$ & 0.285 \\
\hline Digit symbol & $9.51 \pm 3.06$ & $11.07 \pm 2.40$ & 0.008 \\
\hline \multicolumn{4}{|l|}{ Estimated premorbid $1 Q$} \\
\hline Verbal & $110.08 \pm 6.99$ & $112.02 \pm 6.96$ & 0.231 \\
\hline Performance & $110.18 \pm 5.92$ & $111.76 \pm 5.28$ & 0.184 \\
\hline Full scale & $108.96 \pm 6.13$ & $110.29 \pm 4.43$ & 0.180 \\
\hline
\end{tabular}

Values are presented as mean \pm standard deviation. 
Table 4. Comparisons of MMPI and BPRS scores in the intellectual subgroups of schizophrenia participants

\begin{tabular}{|c|c|c|c|}
\hline \multirow[b]{2}{*}{ Variable } & \multicolumn{2}{|c|}{ Group } & \multirow[b]{2}{*}{$p$ value } \\
\hline & $\begin{array}{l}\text { Severely } \\
\text { impaired } \\
(n=25)\end{array}$ & $\begin{array}{l}\text { Mildly } \\
\text { impaired } \\
(n=19)\end{array}$ & \\
\hline \multicolumn{4}{|l|}{ MMPI } \\
\hline \multicolumn{4}{|l|}{ Validity scales } \\
\hline Lie & $46.64 \pm 8.86$ & $47.79 \pm 11.97$ & 0.716 \\
\hline $\mathrm{F}$ & $56.76 \pm 11.81$ & $51.05 \pm 8.89$ & 0.086 \\
\hline K & $46.08 \pm 11.79$ & $53.58 \pm 11.25$ & 0.039 \\
\hline \multicolumn{4}{|l|}{ Clinical scales } \\
\hline Hypochondriasis & $50.00 \pm 11.87$ & $53.37 \pm 9.50$ & 0.537 \\
\hline Depression & $53.36 \pm 13.76$ & $54.11 \pm 13.28$ & 0.858 \\
\hline Hysteria & $50.16 \pm 9.81$ & $51.79 \pm 6.67$ & 0.316 \\
\hline Psychopathic deviate & $52.48 \pm 8.05$ & $61.26 \pm 10.38$ & 0.003 \\
\hline Masculinity-femininity & $47.96 \pm 9.96$ & $52.26 \pm 9.67$ & 0.158 \\
\hline Paranoia & $65.80 \pm 14.14$ & $59.00 \pm 15.86$ & 0.141 \\
\hline Psychasthenia & $61.12 \pm 10.56$ & $56.63 \pm 15.83$ & 0.294 \\
\hline Schizophrenia & $65.72 \pm 9.12$ & $56.37 \pm 11.91$ & 0.005 \\
\hline Hypomania & $56.32 \pm 13.28$ & $51.11 \pm 11.20$ & 0.175 \\
\hline Social introversion & $53.28 \pm 9.33$ & $55.00 \pm 14.12$ & 0.629 \\
\hline BPRS & $58.48 \pm 13.33$ & $45.78 \pm 9.61$ & 0.001 \\
\hline
\end{tabular}

Values are presented as mean \pm standard deviation.

MMPI, Minnesota Multiple Personality Inventory; BPRS, Brief Psychiatric Rating Scale.

higher F, Mf, $\mathrm{Pa}$, and Sc scores, but a significantly lower Pd score, compared with the MIG bipolar disorder participants (Table 5). There was no difference in the BPRS scores of the two groups.

\section{DISCUSSION}

The present study found that the participants with schizophrenia performed significantly poorer on the VIQ, PIQ, and full-scale IQ measures compared with the participants with bipolar disorder.

Only a few studies have directly compared the IQ levels of schizophrenia patients with those of bipolar disorder patients; the majority of studies have compared schizophrenic patients against the general population. A review of 28 studies encompassing more than 1,000 adults with schizophrenia concluded that individuals with schizophrenia performed slightly below the average of the general population on tests of intellectual function and that they exhibited a mean IQ deficit of approximately 10 points. ${ }^{13)}$ Pollack et al. ${ }^{14)}$ compared the intellectual function of 27 adults with schizophrenia with that of their nearest-age siblings and found a mean deficit of 8 IQ points in those with schizophrenic. More recently, Nelson et al. ${ }^{15)}$ found that the mean IQ of 63 patients with schizophrenia was significantly lower than that of the normal population.

In the present study, participants with schizophrenia
Table 5. Comparisons of MMPI and BPRS scores in the intellectual subgroups of bipolar disorder participants

\begin{tabular}{llll}
\cline { 2 - 3 } Variable & \multicolumn{2}{c}{ Group } & \\
\cline { 2 - 3 } & \multicolumn{1}{c}{$\begin{array}{c}\text { Severely } \\
\text { impaired } \\
(n=25)\end{array}$} & $\begin{array}{c}\text { Mildly } \\
\text { impaired } \\
(n=19)\end{array}$ & \\
\hline MMPI & & & \\
Validity scales & & & \\
Lie & $47.31 \pm 8.45$ & $44.24 \pm 7.24$ & 0.235 \\
F & $54.54 \pm 11.25$ & $45.93 \pm 6.55$ & 0.003 \\
K & $43.69 \pm 8.55$ & $47.21 \pm 6.99$ & 0.167 \\
Clinical scales & & & \\
Hypochondriasis & $50.38 \pm 8.50$ & $54.62 \pm 11.55$ & 0.584 \\
Depression & $54.15 \pm 17.18$ & $59.07 \pm 20.17$ & 0.450 \\
Hysteria & $52.54 \pm 7.67$ & $50.97 \pm 11.55$ & 0.139 \\
Psychopathic deviate & $51.54 \pm 8.14$ & $60.28 \pm 7.95$ & 0.002 \\
Masculinity-femininity & $57.08 \pm 12.04$ & $47.52 \pm 9.85$ & 0.006 \\
Paranoia & $63.23 \pm 8.30$ & $54.34 \pm 9.37$ & 0.005 \\
Psychasthenia & $64.62 \pm 9.88$ & $57.34 \pm 14.06$ & 0.100 \\
Schizophrenia & $63.38 \pm 7.86$ & $54.52 \pm 9.07$ & 0.004 \\
Hypomania & $54.85 \pm 10.68$ & $54.24 \pm 11.25$ & 0.871 \\
Social introversion & $51.46 \pm 11.57$ & $53.97 \pm 15.27$ & 0.602 \\
BPRS & $54.15 \pm 15.07$ & $51.14 \pm 12.69$ & 0.506 \\
\hline
\end{tabular}

Values are presented as mean \pm standard deviation.

MMPI, Minnesota Multiple Personality Inventory; BPRS, Brief Psychiatric Rating Scale.

showed significantly lower scores on the vocabulary, comprehension, block design, and digit symbol K-WAIS subtests compared with participants with bipolar disorder. The digit symbol subtest appears to be the most sensitive marker of cognitive impairment in patients with schizophrenia $^{16)}$ and in the nonpsychotic relatives of patients with schizophrenia. ${ }^{17)}$ This study also indicated that performance levels on this task can differentiate siblings at high risk of schizophrenia who later became psychotic from those who do not. Taken together, these findings indicate that impaired performance on the digit symbol subtest is a stable trait in patients with schizophrenia and likely reflects the existence of abnormal cognitive processing that is central to the disorder. ${ }^{16)}$ This impairment is present at the onset of psychosis, even as performance remains intact on other tests of general intellectual ability. ${ }^{18)}$ The digit symbol subtest is impervious to practice effects and remains unaffected by the administration of antipsychotic medications during the first year of illness. ${ }^{18)}$ Furthermore, the comprehension and digit symbol subtests are closely associated with functional outcomes in patients with schizophrenia and in those with first-episode psychosis. ${ }^{19)}$ Consistent with these findings, previous studies have found no differences on the information and digit span subtests between the two disorders. ${ }^{18,19)}$ This finding indicates that even though patients with schizophrenia experience overall cognitive impairment, they may have crys- 
tallized intelligence that is largely not affected by the illness. $^{19)}$

In the present study, $52.9 \%$ of participants with schizophrenia and $31 \%$ of participants with bipolar disorder appeared to experience a severe intellectual decline. There were significantly more SIG schizophrenia than SIG bipolar disorder participants, which is consistent with the findings of previous studies. ${ }^{20,21)}$ It appears that patients with schizophrenia and those with bipolar disorder share a similar cognitive impairment profile, with varying degrees of deficits. $^{20)}$

In the present study, the SIG schizophrenia participants had significantly higher scores on the Sc scales of the MMPI and BPRS compared with the MIG schizophrenia patients. These differences suggest that the SIG participants exhibited more severe psychopathology than do MIG participants with schizophrenia. The Sc scale measures psychosis-related symptoms, poor judgment, unconventional thoughts or behaviors, possible overvalued ideas, feelings of isolation, and alienation. ${ }^{22)}$ Similarly, the BPRS also reflects the severity of psychotic symptoms. ${ }^{23)}$ The present study identified a moderate simultaneous elevation of scores on the $\mathrm{Sc}$ and $\mathrm{Pa}$ scales in the SIG participants. The $\mathrm{Pa}$ subscale is associated with rigid thinking, interpersonal sensitivity, and suspiciousness. ${ }^{22)}$ Patients with high scores on the $\mathrm{Sc}$ and $\mathrm{Pa}$ scales tend to have less social interest, to be more likely to be unfriendly with others, more emotionally withdrawn, more conceptually disorganized, and more suspicious, and to have more hallucinatory behaviors and a greater degree of unusual thought content. ${ }^{24)}$ These findings suggest higher levels of these characteristics in SIG than in MIG participants.

In the present study, the SIG bipolar disorder participants had significantly higher scores on the Mf, $\mathrm{Pa}$, and Sc scales than the MIG participants did, which suggests greater symptom severity in the SIG subjects. Accordingly, women with elevated Mf scores may have difficulty appropriately channeling aggressive impulses. ${ }^{25)}$

For both schizophrenia and bipolar disorder, the $\mathrm{Pd}$ scores of the SIG participants were lower than those of the MIG participants. The Pd scale measures impulsivity, frustration tolerance, and risk taking. ${ }^{25)}$ A low Pd score may indicate suppressed aggressive and assertive tendencies as well as low sexual interest. ${ }^{25)}$

SIG patients with elevated Pd and Sc scores may have marginal psychological adjustment during the course of schizophrenia or bipolar disorder. ${ }^{24)}$ These patients tend to have intense feelings of inferiority and insecurity, to be suspicious and distrustful of others, to avoid deep emotional ties, and to present with thought disorders, hallucinations, delusions, hostility, and a lack of insight. ${ }^{24)}$ Additionally, poor judgment is typical of these patients. ${ }^{24)}$ These tendencies were more likely to be prominent in the SIG participants from both groups in the present study, indicating that the participants with severe cognitive decline exhibited more psychotic symptoms, a greater degree of thought disorder, impaired reality testing, and difficulty adjusting. Thus, more severe psychopathology was associated with apparent intellectual decline.

The findings of the present study revealed a significant discrepancy between the estimated premorbid levels of cognitive function and current cognitive function. Moreover, this discrepancy was correlated with the severity of psychopathology in patients from both groups. The present results are consistent with those of a previous study of 513 inpatients with psychotic disorders, which found that a larger difference between estimated premorbid and current IQ levels predicted a greater number of psychotic indicators. $^{26)}$

There are several limitations to the present study. First, the design of this study was not longitudinal, and the premorbid IQ scores were not directly obtained. A second limitation is that the effects of psychiatric medications on the cognitive function of the participants cannot be ruled out; it is possible that the cognitive deficits observed in the present study were due to the effects of medication. Finally, no normal control group was available, and therefore, it was not possible to determine whether results for these patients deviated from the norm.

\section{- Acknowledgments}

This work was supported by the research grant of the Jeju National University in 2011.

\section{REFERENCES}

1. Heinrichs RW, Zakzanis KK. Neurocognitive deficit in schizophrenia: a quantitative review of the evidence. Neuropsychology 1998;12:426-445.

2. Green MF. What are the functional consequences of neurocognitive deficits in schizophrenia? Am J Psychiatry 1996;153:321-330.

3. McClellan J, Prezbindowski A, Breiger D, McCurry C. Neuropsychological functioning in early onset psychotic disorders. Schizophr Res 2004;68:21-26.

4. Martínez-Arán A, Vieta E, Colom F, Torrent C, Sánchez-Moreno J, Reinares M, et al. Cognitive impairment in euthymic bipolar patients: implications for clinical and functional outcome. Bipolar Disord 2004;6:224-232.

5. Wingo AP, Harvey PD, Baldessarini RJ. Neurocognitive impairment in bipolar disorder patients: functional implications. Bipolar Disord 2009;11:113-125. 
6. Altshuler L, Tekell J, Biswas K, Kilbourne AM, Evans D, Tang $\mathrm{D}$, et al. Executive function and employment status among veterans with bipolar disorder. Psychiatr Serv 2007;58:1441-1447.

7. Ventura J, Hellemann GS, Thames AD, Koellner V, Nuechterlein KH. Symptoms as mediators of the relationship between neurocognition and functional outcome in schizophrenia: a meta-analysis. Schizophr Res 2009;113: 189-199.

8. Albus M, Hubmann W, Wahlheim C, Sobizack N, Franz U, Mohr F. Contrasts in neuropsychological test profile between patients with first-episode schizophrenia and first-episode affective disorders. Acta Psychiatr Scand 1996;94:87-93.

9. Yeom TH, Park YS, Oh KJ, Kim JK, Lee YH. KoreaWechsler Adult Intelligence Scale. Seoul:Korea Guidance;1 992.

10. Kim HK. Estimating premorbid intelligence: After Year 2001. Korean J Clin Psychol 2001;20:155-164.

11. Kim YH, Kim JH, Kim JK, Rho MR, Shin DK, Yum TH, et al. Manual for MMPI. Seoul: Korean Guidance;1989.

12. Bell M, Milstein R, Beam-Goulet J, Lysaker P, Cicchetti D. The Positive and Negative Syndrome Scale and the Brief Psychiatric Rating Scale. Reliability, comparability, and predictive validity. J Nerv Ment Dis 1992;180:723-728.

13. Payne R. Cognitive abnormalities. In: Eysenck HJ, editor. Handbook of abnormal psychology: an experimental approach. London:Pitman Medical Pub;1960. p.193-261.

14. Pollack M, Woerner MG, Klein DF. A comparison of childhood characteristics of schizophrenics, personality disorders and their characteristics of schizophrenics, personality disorders and their siblings. In: Roff M, Ricks DF, Pollack M; Society for Life History Research in Psychopathology (Los Angeles, CA), editors. Life history research in psychopathology. Minneapolis, MN:University of Minnesota Press;1970. p.208-225

15. Nelson HE, Pantelis C, Carruthers K, Speller J, Baxendale $\mathrm{S}$, Barnes TR. Cognitive functioning and symptomatology in chronic schizophrenia. Psychol Med 1990;20:357-365.

16. Dickinson D, Ramsey ME, Gold JM. Overlooking the obvious: a meta-analytic comparison of digit symbol coding tasks and other cognitive measures in schizophrenia. Arch Gen Psychiatry 2007;64:532-542.

17. Niendam TA, Bearden CE, Rosso IM, Sanchez LE, Hadley $\mathrm{T}$, Nuechterlein $\mathrm{KH}$, et al. A prospective study of childhood neurocognitive functioning in schizophrenic patients and their siblings. Am J Psychiatry 2003;160:2060-2062.

18. Leeson VC, Barnes TR, Harrison M, Matheson E, Harrison I, Mutsatsa SH, et al. The relationship between IQ, memory, executive function, and processing speed in recent-onset psychosis: 1-year stability and clinical outcome. Schizophr Bull 2010;36:400-409.

19. Fujino H, Sumiyoshi C, Sumiyoshi T, Yasuda Y, Yamamori $\mathrm{H}$, Ohi $\mathrm{K}$, et al. Performance on the Wechsler Adult Intelligence Scale-III in Japanese patients with schizophrenia. Psychiatry Clin Neurosci 2014;68:534-541.

20. Vöhringer PA, Barroilhet SA, Amerio A, Reale ML, Alvear $\mathrm{K}$, Vergne $\mathrm{D}$, et al. Cognitive impairment in bipolar disorder and schizophrenia: a systematic review. Front Psychiatry 2013;4:87.

21. Bora E. Developmental trajectory of cognitive impairment in bipolar disorder: Comparison with schizophrenia. Eur Neuropsychopharmacol 2015;25:158-168.

22. Demidenko N, Grof P, Alda M, Deshauer D, Duffy A. MMPI as a measure of subthreshold and residual psychopathology among the offspring of lithium responsive and non-responsive bipolar parents. Bipolar Disord 2004;6: 323-328.

23. Grisso T, Appelbaum PS. The MacArthur Treatment Competence Study. III: Abilities of patients to consent to psychiatric and medical treatments. Law Hum Behav 1995; 19:149-174.

24. Duckworth JC, Anderson WP. MMPI interpretation manual for counselors and clinicians. Bristor, PA:Accelerated Development Inc;1986.

25. Graham JR. The MMPI: A practical guide. New York:Oxford University Press; 1987.

26. Bilginer L, DeLuca V, Pogge DL, Stokes JS, Harvey PD. Intellectual functioning in adolescents with indicators of psychosis: evidence for decline in functioning related to number of psychotic features? J Neuropsychiatry Clin Neurosci 2005;17:106-113. 